PROCEEDINGS OF THE

AMERICAN MATHEMATICAL SOCIETY

Volume 128, Number 12, Pages 3711-3714

S 0002-9939(00)05589-1

Article electronically published on June 7, 2000

\title{
OPENNESS OF INDUCED PROJECTIONS
}

\author{
JANUSZ J. CHARATONIK, WŁODZIMIERZ J. CHARATONIK, AND \\ ALEJANDRO ILLANES
}

(Communicated by Alan Dow)

\begin{abstract}
For continua $X$ and $Y$ it is shown that if the projection $f: X \times$ $Y \rightarrow X$ has its induced mapping $C(f)$ open, then $X$ is $C^{*}$-smooth. As a corollary, a characterization of dendrites in these terms is obtained.
\end{abstract}

All spaces considered in this paper are assumed to be metric. A mapping means a continuous function. To exclude some trivial statements we assume that all considered mappings are not constant. A continuum means a compact connected space. Given a continuum $X$ with a metric $d$, we let $2^{X}$ denote the hyperspace of all nonempty closed subsets of $X$ equipped with the Hausdorff metric $H$ defined by

$$
H(A, B)=\max \{\sup \{d(a, B): a \in A\}, \sup \{d(b, A): b \in B\}\}
$$

(see e.g. [6, (0.1), p. 1, and (0.12), p. 10]). Further, we denote by $C(X)$ the hyperspace of all subcontinua of $X$, i.e., of all connected elements of $2^{X}$. The reader is referred to Nadler's book [6] for needed information on the structure of hyperspaces.

Given a mapping $f: X \rightarrow Y$ between continua $X$ and $Y$, we consider mappings (called the induced ones)

$$
2^{f}: 2^{X} \rightarrow 2^{Y} \quad \text { and } \quad C(f): C(X) \rightarrow C(Y)
$$

defined by

$2^{f}(A)=f(A)$ for every $A \in 2^{X} \quad$ and $\quad C(f)(A)=f(A)$ for every $A \in C(X)$.

A mapping $f: X \rightarrow Y$ between spaces $X$ and $Y$ is said to be open provided the image of an open subset of the domain is open in the range. The following results concerning induced mappings for the class of open mappings are known (see [4] Theorem 4.3]; compare also [3, Theorem 3.2]).

1. Statement. Let a surjective mapping $f: X \rightarrow Y$ between continua $X$ and $Y$ be given. Consider the following conditions:

(1.1) $f: X \rightarrow Y$ is open;

(1.2) $C(f): C(X) \rightarrow C(Y)$ is open;

(1.3) $2^{f}: 2^{X} \rightarrow 2^{Y}$ is open.

Then (1.1) and (1.3) are equivalent, and each of them is implied by (1.2).

Received by the editors February 2, 1999.

2000 Mathematics Subject Classification. Primary 54B20, 54E40, 54F15, 54F50, 54F65.

Key words and phrases. $C^{*}$-smooth, continuum, dendrite, induced mapping, open, projection.

(C)2000 American Mathematical Society 
An example is known [4, Section 4] of open surjective mappings $f: X \rightarrow Y$ between locally connected continua $X$ and $Y$ such that the induced mapping $C(f)$ : $C(X) \rightarrow C(Y)$ is not open.

A continuum, the intersection of every two subcontinua of which is connected, is said to be hereditarily unicoherent. A continuum is called a dendroid provided that it is hereditarily unicoherent and arcwise connected. Given points $a$ and $b$ in a dendroid $X$, we denote by $a b$ the (unique) arc in $X$ joining these points.

The following result has been proved in [1, Theorem 21].

2. Theorem. Let $X$ and $Y$ be nondegenerate continua, and let $f: X \times Y \rightarrow X$ denote the natural projection. If $C(f)$ is open, then $X$ is hereditarily unicoherent.

It is known that the opposite implication is not true (see [1, Example 22]). The aim of the paper is to present further results in this direction.

Given a (metric) space $X$ we denote by $d_{X}$ the metric on $X$, and by $B_{X}(p, \varepsilon)$ the (open) ball in $X$ centered at a point $p \in X$ and having the radius $\varepsilon$. Given a subset $A \subset X$, we define $N_{X}(A, \varepsilon)=\bigcup\left\{B_{X}(a, \varepsilon): a \in A\right\}$, and we use the symbol $\operatorname{cl}_{X}(A)$ to denote the closure of $A$ in $X$. The symbol $\mathbb{N}$ stands for the set of all positive integers.

Let $X$ be a continuum. Define $C^{*}: C(X) \rightarrow C(C(X))$ by $C^{*}(A)=C(A)$. It is known that for any continuum $X$ the function $C^{*}$ is upper semicontinuous (see [6. Theorem 15.2, p. 514]), and it is continuous on a dense $G_{\delta}$ subset of $C(X)$ (see [6. Corollary 15.3, p. 515]). A continuum $X$ is said to be $C^{*}$-smooth at $A \in C(X)$ provided that the function $C^{*}$ is continuous at $A$. A continuum $X$ is said to be $C^{*}$-smooth provided that the function $C^{*}$ is continuous on $C(X)$, i.e., at each $A \in C(X)$ (see 6. Definition 5.15, p. 517]). Each arclike continuum is $C^{*}$-smooth, (6. Theorem 15.13, p. 525]). $C^{*}$-smoothness implies hereditary unicoherence (see [2, Corollary 3.4, p. 203] and [6, Note 1, p. 530]). Thus each arcwise connected $C^{*}$-smooth continuum is a dendroid (see [6, Theorem 15.19, p. 528]). Further, a locally connected continuum is $C^{*}$-smooth if and only if it is a dendrite (see [6. Theorem 15.11, p. 522]).

3. Lemma. Let $X$ be a nondegenerate continuum, and let $\varepsilon>0$ be given. Then there is a finite sequence of subcontinua $D_{0} \subset D_{1} \subset \ldots \subset D_{m}$ of $X$ and there is an $\varepsilon$-net $\left\{a_{1}, \ldots, a_{m}\right\}$ in $X$ such that $a_{i} \in D_{i} \backslash D_{i-1}$ for each $i \in\{1, \ldots, m\}$.

Proof. Let $\left\{b_{1}, \ldots, b_{m}\right\}$ be an $\frac{\varepsilon}{2}$-net in $X$. Fix a point $b \in X \backslash\left\{b_{1}, \ldots, b_{m}\right\}$. Let $\alpha:[0,1] \rightarrow C(X)$ be an order arc from $\{b\}$ to $X$; that is, a mapping such that $\alpha(0)=\{b\}, \alpha(1)=X$ and, if $s<t$, then $\alpha(s) \subsetneq \alpha(t)$ (for the existence of order arcs see [6, Theorem 1.8, p. 59]). Let $t_{0}>0$ be such that $\alpha\left(t_{0}\right) \cap\left\{b_{1}, \ldots, b_{m}\right\}=\emptyset$. Define $D_{0}=\alpha\left(t_{0}\right)$.

Let $s_{1}=\min \left\{t \in[0,1]: \alpha(t) \cap\left\{b_{1}, \ldots, b_{m}\right\} \neq \emptyset\right\}$. We may assume that $b_{1} \in$ $\alpha\left(s_{1}\right)$. Note that $t_{0}<s_{1}$. Consider the set $E=\left(X \backslash B_{X}\left(b_{1}, \frac{\varepsilon}{2}\right)\right) \cup D_{0}$. Then $E$ is a closed subset of $X$ and $b_{1} \in X \backslash E$. Thus there exists $t_{1} \in\left(t_{0}, s_{1}\right)$ such that $\alpha\left(t_{1}\right)$ is not contained in $E$. Choose a point $a_{1} \in \alpha\left(t_{1}\right) \backslash E$. Observe that $a_{1} \in B_{X}\left(b_{1}, \frac{\varepsilon}{2}\right) \backslash D_{0}$. Define $D_{1}=\alpha\left(t_{1}\right)$.

Let $s_{2}=\min \left\{t \in[0,1]: \alpha(t) \cap\left\{b_{2}, \ldots, b_{m}\right\} \neq \emptyset\right\}$. We may assume that $b_{2} \in$ $\alpha\left(s_{2}\right)$. Note that $t_{1}<s_{2}$. Proceeding as in the paragraph above it is possible to find a number $t_{2} \in\left(t_{1}, s_{2}\right)$ and a point $a_{2} \in \alpha\left(t_{2}\right) \cap B_{X}\left(b_{2}, \frac{\varepsilon}{2}\right) \backslash D_{1}$. Define $D_{2}=\alpha\left(t_{2}\right)$.

Following this procedure we can find points $a_{1}, a_{2}, \ldots, a_{m}$ in $X$ and numbers $0<t_{0}<t_{1}<t_{2}<\cdots<t_{m}<1$ such that for each $i \in\{1, \ldots, m\}$ we have 
$a_{i} \in \alpha\left(t_{i}\right) \backslash \alpha\left(t_{i-1}\right)$ and $d\left(a_{i}, b_{i}\right)<\frac{\varepsilon}{2}$. Defining $D_{i}=\alpha\left(t_{i}\right)$ for each $i \in\{0,1, \ldots, m\}$ we see that the continua $D_{0}, D_{1}, \ldots, D_{m}$ and the points $a_{1}, \ldots, a_{m}$ satisfy the required conditions. The proof is complete.

Let $n \in \mathbb{N}$. A finite sequence of $n$ sets $L_{1}, \ldots, L_{n}$ is called a chain provided that $L_{i} \cap L_{j} \neq \emptyset$ if and only if $|i-j| \leq 1$. Elements $L_{i}$ of the chain are called its links.

4. Theorem. Let $X$ and $Y$ be nondegenerate continua, and let $f: X \times Y \rightarrow X$ denote the natural projection. If $C(f)$ is open, then $X$ is $C^{*}$-smooth.

Proof. Assume the contrary. Let $\mathcal{A}=\operatorname{Lim} C\left(A_{n}\right) \subsetneq C(A)$ for a sequence of subcontinua $A_{n}$ of $X$ converging to a continuum $A$, and take $K \in C(A) \backslash \mathcal{A}$. Let $\varepsilon>0$ be such that $B_{C(X)}(K, 2 \varepsilon) \cap C\left(A_{i}\right)=\emptyset$ for each $i \in \mathbb{N}$.

Let $D_{0}, D_{1}, \ldots, D_{m}$ and $\left\{a_{1}, \ldots, a_{m}\right\}$ be as in Lemma 3 for the continuum $K$. Choose subcontinua $E_{0} \subset E_{1} \subset \ldots \subset E_{m}$ of $Y$ and points $b_{i} \in E_{i} \backslash E_{i-1}$. Fix points $a_{0} \in D_{0}$ and $b_{0} \in E_{0}$.

Note that the sequence $\left\{a_{0}\right\} \times E_{0}, D_{1} \times\left\{b_{0}\right\},\left\{a_{1}\right\} \times E_{1}, D_{2} \times\left\{b_{1}\right\},\left\{a_{2}\right\} \times$ $E_{2}, \ldots, D_{m} \times\left\{b_{m-1}\right\},\left\{a_{m}\right\} \times E_{m}, A \times\left\{b_{m}\right\}$ is a chain. Let $P$ be the union of the chain, i.e.,

$$
\begin{aligned}
P=\left(A \times\left\{b_{m}\right\}\right) \cup\left(D_{1} \times\left\{b_{0}\right\}\right) \cup\left(D_{2} \times\left\{b_{1}\right\}\right) \cup \cdots \cup\left(D_{m} \times\left\{b_{m-1}\right\}\right) \\
\cup\left(\left\{a_{0}\right\} \times E_{0}\right) \cup\left(\left\{a_{1}\right\} \times E_{1}\right) \cup \cdots \cup\left(\left\{a_{m}\right\} \times E_{m}\right),
\end{aligned}
$$

and note that $P$ is a subcontinuum of $X \times Y$ and that $C(f)(P)=A$.

Choose a number $\eta$ with $0<\eta<\varepsilon$ satisfying the two conditions

$$
\begin{array}{ccc}
N_{X}\left(D_{i}, \eta\right) \cap B_{X}\left(a_{j}, \eta\right)=\emptyset & \text { for } & 0 \leq i<j, \\
N_{Y}\left(E_{i}, \eta\right) \cap B_{Y}\left(b_{j}, \eta\right)=\emptyset & \text { for } & 0 \leq i<j .
\end{array}
$$

It follows that the sequence

$$
\begin{aligned}
N_{X \times Y}\left(\left\{a_{0}\right\} \times E_{0}, \eta\right), N_{X \times Y}\left(D_{1} \times\left\{b_{0}\right\}, \eta\right), N_{X \times Y}\left(\left\{a_{1}\right\} \times E_{1}, \eta\right), & \\
& N_{X \times Y}\left(D_{2} \times\left\{b_{1}\right\}, \eta\right), N_{X \times Y}\left(\left\{a_{2}\right\} \times E_{2}, \eta\right), \ldots, \\
& N_{X \times Y}\left(D_{m} \times\left\{b_{m-1}\right\}, \eta\right), N_{X \times Y}\left(\left\{a_{m}\right\} \times E_{m}, \eta\right), N_{X \times Y}\left(A \times\left\{b_{m}\right\}, \eta\right)
\end{aligned}
$$

is a chain. By interiority of $C(f)$ at $P$ there is a $\delta>0$ such that $B_{C(X)}(f(P), \delta) \subset$ $C(f)\left(B_{C(X \times Y)}(P, \eta)\right)$. Let $k \in \mathbb{N}$ be such that $H\left(A_{k}, A\right)<\delta$. Then there is a continuum $Q \subset X \times Y$ such that $H(P, Q)<\eta$ and $f(Q)=A_{k}$. Take a point $q \in Q$ such that $d_{X \times Y}\left(q,\left(a_{0}, b_{0}\right)\right)<\eta$. Let $L$ be the component of $Q \backslash N_{X \times Y}\left(A \times\left\{b_{m}\right\}, \eta\right)$ containing $q$. By the Janiszewski theorem (known also as the boundary bumping theorem; see e.g. [5, §47, III, Theorems 1 and 2, p. 172] and compare [6, 20.1-20.3, p. 625-626]) there is a point $r \in L \cap \operatorname{cl} N_{X \times Y}\left(A \times\left\{b_{m}\right\}, \eta\right)$. Thus $L$ intersects the first and the closure of the last link of the chain (4.1), and it is contained in the union of all links of this chain. Consequently, $L$ intersects each intermediate link of the chain.

Let $q_{i} \in L \cap N_{X \times Y}\left(\left\{a_{i}\right\} \times E_{i}, \eta\right)$. Note that $d_{X}\left(f\left(q_{i}\right), a_{i}\right)<\eta$. Thus we have $f(L) \subset N_{X}(K, \eta) \subset N_{X}(K, 2 \varepsilon)$ and

$$
\begin{aligned}
K \subset N_{X}\left(\left\{a_{1}, \ldots, a_{m}\right\}, \varepsilon\right) \subset N_{X}\left(\left\{f\left(q_{1}\right), \ldots, f\left(q_{m}\right)\right\}, \varepsilon+\eta\right) & \\
& \subset N_{X}(f(L), \varepsilon+\eta) \subset N_{X}(f(L), 2 \varepsilon) .
\end{aligned}
$$

It follows that $H(K, f(L))<2 \varepsilon$ and $f(L) \in C\left(A_{k}\right)$, contrary to the definition of $\varepsilon$. The proof is then complete. 
5. Corollary. For a fixed continuum $X$ let $f_{Y}: X \times Y \rightarrow X$ denote the natural projection. The following conditions are equivalent for a locally connected continuum $X$ :

(5.1) $X$ is a dendrite;

(5.2) for each continuum $Y$ the induced mapping $C\left(f_{Y}\right)$ is open;

(5.3) the induced mapping $C\left(f_{[0,1]}\right)$ is open;

(5.4) there exists a continuum $Y$ such that the induced mapping $C\left(f_{Y}\right)$ is open.

Proof. The implication from (5.1) to (5.2) is known from [1, Corollary 35]. The implications $(5.2) \Longrightarrow(5.3) \Longrightarrow(5.4)$ are obvious. Finally (5.4) implies that $X$ is $C^{*}$-smooth according to Theorem 4 , which for locally connected continua is equivalent to be a dendrite (see [6, Theorem 15.11, p. 522]).

The following problem seems to be interesting.

6. Problem. Characterize the continua $X$ for which the converse implication to that of Theorem 4 is true, i.e., the continua $X$ such that the induced mapping $C\left(f_{Y}\right): C(X \times Y) \rightarrow C(X)$ is open for each continuum $Y$. In particular, is $C^{*}-$ smoothness of $X$ sufficient?

\section{REFERENCES}

1. J. J. Charatonik, W. J. Charatonik and A. Illanes, Openness of induced mappings, Topology Appl. 98 (1999), 67-80. CMP 99:17

2. J. Grispolakis, S. B. Nadler, Jr., and E. D. Tymchatyn, Some properties of hyperspaces with applications to continua theory, Canad. J. Math. 31 (1979), 197-210. MR 80j:54029

3. H. Hosokawa, Induced mappings between hyperspaces II, Bull. Tokyo Gakugei Univ. (4) 44 (1992), 1-7. MR 94a:54036

4. H. Hosokawa, Induced mappings on hyperspaces, Tsukuba J. Math. 21 (1997), 239-250. MR 98f:54011

5. K. Kuratowski, Topology, vol. 2, Academic Press and PWN, New York, London and Warszawa, 1968. MR 41:4467

6. S. B. Nadler, Jr., Hyperspaces of sets, M. Dekker, 1978. MR 58:18330

(J. J. Charatonik) Mathematical Institute, University of Wroceaw, Pl. Grunwaldzki 2/4, 50-384 Wroceaw, Poland

E-mail address: jjc@hera.math.uni.wroc.pl

(J. J. Charatonik) Instituto de Matemáticas, UnAm, Circuito Exterior, Ciudad Universitaria, 04510 MéXico, D. F., MÉXiCO

E-mail address: jjc@gauss.matem.unam.mx

(W. J. Charatonik) Mathematical Institute, University of Wroceaw, Pl. Grunwaldzki 2/4, 50-384 Wroceaw, Poland

E-mail address: wjcharat@hera.math.uni.wroc.pl

(W. J. Charatonik) Departamento de Matemáticas, Facultad de Ciencias, UnAm, CirCuito Exterior, Ciudad Universitaria, 04510 México, D. F., México

E-mail address: wjcharat@lya.fciencias.unam.mx

Current address, W. J. Charatonik: Department of Mathematics and Statistics, University of Missouri-Rolla, Rolla, Missouri 65409-0020

(A. Illanes) Instituto de Matemátic as, UnAm, Circuito Exterior, Ciudad UniversiTARIA, 04510 MÉXICO, D. F., MÉXiCO

E-mail address: illanes@gauss.matem.unam.mx 